\title{
The efficacy and clinical outcomes of transarterial embolization in acute massive upper gastrointestinal bleeding: a single-center experience
}

\author{
Mehmet TAHTABASI ${ }^{1}$ (D), Mehmet KOLU ${ }^{2}$ (iD \\ ${ }^{1}$ Department of Radiology, Mehmet Akif Inan Education and Research Hospital, University of Health Sciences, Sanliurfa, Turkey. \\ ${ }^{2}$ Department of Radiology, School of Medicine, Harran University, Sanliurfa, Turkey.
}

Corresponding Author: Mehmet TAHTABASI

E-mail: mehmet.tahtabasi@sbu.edu.tr

Submitted: 25.11.2020 Accepted: 13.02.2021

\section{ABSTRACT}

Objective: To evaluate the efficacy of transarterial embolization (TAE) in the treatment of acute upper gastrointestinal bleeding (UGIB) and identify potential factors affecting the treatment outcome.

Patients and Methods: Eighteen patients with UGIB treated with TAE were included in the study. The demographic data, causes of bleeding, angiography findings, treatments applied, and clinical outcomes were retrospectively analyzed.

Results: Thirteen (72.2\%) patients were male, five (27.8\%) were female, and the mean age was 55 years (range: 33-68 years). A pseudoaneurysm ( $\mathrm{n}=7 ; 38.9 \%)$ was the most common finding, followed by tumor staining $(\mathrm{n}=4 ; 22.2 \%)$. The most common pathology causing bleeding was peptic ulcer disease $(\mathrm{n}=9 ; 50 \%)$. The most commonly embolized artery was the gastroduodenal artery $(\mathrm{n}=11 ; 61.1 \%)$. There were three $(16.7 \%)$ patients with hemobilia due to iatrogenic hepatic artery pseudoaneurysm. In most cases $(n=9 ; 50 \%)$, coils and polyvinyl alcohol particles were used in combination. Technical success was achieved in all patients. The clinical success rate was $78.5 \%$ (11/14 patients) after the exclusion of patients who died for non-UGIB causes $(n=4 ; 22.2 \%)$.

Conclusion: Transarterial embolization is an effective method used with high technical and clinical success rates in the treatment of endoscopy-refractory acute UGIB.

Keywords: Interventional imaging, Nonvariceal upper gastrointestinal bleeding, Endoscopy, Angiography, Embolization

\section{INTRODUCTION}

Acute upper gastrointestinal bleeding (UGIB) is an emergency that can lead to morbidity and mortality if proper treatment is not undertaken promptly [1]. Anatomically, UGIB refers to bleeding originating from the distal esophagus, stomach and duodenum, proximal to the ligament of Treitz. It is a common pathology with an incidence of 50-100 per 100,000 population and a mean disease age of 60-70 years [2]. Peptic ulcer disease (PUD) is the most common cause of non-varicose UGIB, and it less frequently occurs due to arteriovenous malformations, such as benign and malignant tumors, gastritis, ischemia, Dieulafoy's lesions, Mallory-Weiss syndrome, trauma, and iatrogenic causes [3]. A rapid and accurate diagnosis (localization and etiology) should be made in order to effectively treat acute massive UGIB. Following patient stabilization, endoscopy is usually performed to detect active bleeding and achieve hemostasis, but this primary hemostatic procedure fails in $10-30 \%$ of patients. If bleeding ( $1 \mathrm{ml} / \mathrm{min})$ still continues after endoscopy or primary hemostasis fails despite endoscopic techniques, a more aggressive treatment with transarterial embolization (TAE) or surgical intervention is required. TAE has replaced the surgical procedure in recent years and has been shown to be effective in controlling bleeding and reducing mortality $[3,4]$. The current study aimed to evaluate the safety and efficacy of TAE in the treatment of acute UGIB due to non-varicose causes and to identify potential factors affecting the treatment outcome.

How to cite this article: Tahtabasi M, Kolu M. The efficacy and clinical outcomes of transarterial embolization in acute massive upper gastrointestinal bleeding: a single-center experience. Marmara Med J 2021; 34(2):180-188. doi: 10.5472/marumj.944254 


\section{PATIENTS and METHODS}

\section{Patient population}

For this study, the clinical records of 37 patients treated for acute massive UGIB between March 2018 and October 2019 at our center were retrospectively examined. Patients treated with medical $(n=6)$, surgical $(n=5)$ and endoscopic $(n=8)$ methods were excluded. As a result, a total of 18 patients treated in the interventional radiology unit with endovascular methods were included in the study. Ethics committee approval was obtained for this single-center retrospective study (approval number: 2020/ 8-12).

During the angiography procedure, all patients had active massive UGIB that did not respond to medical and endoscopic treatment. The study group consisted of 13 men and five women, and the mean age was 55 years (range: 33-68 years).

\section{Clinical data and definitions}

The demographic characteristics, laboratory findings, comorbidities, bleeding pathology, rebleeding, clinical findings, and mortality rates were obtained from the electronic medical records of the patients. The shock index (heart rate/systolic blood pressure) indicating the hemodynamic instability of each patient was recorded. A shock index of $>0.9$ was defined as hypovolemic shock [5]. The hemoglobin concentration at the time of presentation and the number of blood units transfused before embolization were measured. The presence of hemodynamic instability and the need for at least four units of blood within 24 hours was evaluated as acute massive UGIB [3]. The presence of one or more of the following criteria was considered to indicate coagulopathy: International normalized ratio $($ INR) $>1.6$, partial thromboplastin time $>45 \mathrm{sec}$, or prothrombin time $>13.5$ seconds. The patients with a platelet count of $<50,000 / \mathrm{mm}^{3}$ were diagnosed with thrombocytopenia [3]. A pre-angiographic investigation was accepted as endoscopy or computed tomography angiography (CTA) performed after the onset of acute UGIB and seven days before the TAE intervention. All patients underwent endoscopy and CTA before angiography.

\section{Angiography and TAE}

Selective angiographies were performed in the interventional radiology unit by an interventional radiologist (IR) with a minimum of five years of experience in endovascular procedures. All patients signed an informed consent form prior to interventional treatment. After the patients were placed on the operation table for interventional treatment, their blood pressure, oxygen saturation, and heart and respiratory rates were monitored. The right inguinal area was disinfected, and the procedure was started by covering the location with a surgical towel. With the patient under local anesthesia, the common femoral artery was punctured with a retrograde technique using an 18-gauge single-wall needle under ultrasound guidance. Using the Seldinger technique, a 5-French (Fr) vascular sheath was placed over a 0.035 -inch guide-wire. A long femoral guiding sheath (6 Fr - 45 cm Destination ${ }^{\circledR}$ Terumo, Tokyo, Japan) was used in cases where catheterization was difficult due to tortuous arteries. Depending on the bleeding site identified on CTA images, the celiac truncus and the superior mesenteric artery (SMA) were selectively catheterized using 4 -Fr or 5-Fr angiographic catheters (Simmon-1 or Cobra-2; Cordis, Miami Lakes, FL, USA). Angiography was performed to confirm bleeding, localize the source (active contrast extravasation, contrast blushing, or tumor bleeding), or to detect pseudoaneurysms. Then, the target artery was superselectively catheterized using a microcatheter (1.7-2.1 Fr Echelon, Medtronic, USA; 2.7 Fr Progreat ${ }^{\oplus}$, Terumo, Tokyo, Japan). The appropriate embolic agent was selected depending on the patient and administered by the IR to the patient through the microcatheter. The materials used for embolization were 0.018-inch platinum microcoils (Boston Scientific, Ireland) or polyvinyl alcohol (PVA) particles (Contour TM; Boston Scientific), or a combination thereof. The sizes of the PVA particles used were 355-500, 500710 and $710-1,000$ microns. In addition, in the treatment of pseudoaneurysms, a covered stent-graft was placed using a 60 $\mathrm{cm} 8 \mathrm{Fr}$ vascular sheath (Arrow, Arrow International, USA) and a 0.014 inch guide-wire (Jostent, Graftmaster, Coronary stent graft, Germany).

Finally, a post-embolization angiography was performed to confirm the technical success of the procedure and reevaluate the presence of other possible bleeding. The angiographic data were obtained by examining the Picture Archiving and Communication System of the hospital. The bleeding focus associated with the artery feeding the bleeding area and positive angiographic findings were recorded for each patient.

\section{Technical and clinical endpoints}

Based on angiographic findings, technical success was defined as the disappearance of contrast extravasation immediately after embolization, loss of flow in the arteries or their branches feeding the bleeding focus, and disappearance of the filling of the pseudoaneurysm causing bleeding, and technical failure as the spontaneous cessation of bleeding as a result of arterial spasm during catheter advancement or the bleeding artery not being selectively catheterized and embolized. Clinical success was defined as the improvement of the signs and symptoms of bleeding, including hemoglobin level and blood pressure within 30 days following TAE, and clinical failure as persistent or recurrent active bleeding that occurred within 30 days following the procedure, requiring surgical intervention and/or resulting in mortality $[1,6]$.

\section{Short-term follow-up}

In the short-term follow-up of the patients, post-procedural persistent bleeding, early recurrent bleeding within the first three days, and 30-day mortality rates were recorded. It was also determined whether a second intervention, such as surgery or TAE was performed after the procedure. 


\section{Statistical Analysis}

All analyses were performed using SPSS software v. 22.0 (IBM SPSS Statistics Version 22.0. Armonk, NY: IBM Corp.). The variables were divided into two groups as categorical and continuous. Categorical variables were expressed as numbers and percentages, and continuous variables as mean and range values. Technical success, clinical success, technical failure, and clinical failure were calculated as percentages. Categorical variables were compared with Fisher's exact test. The statistical significance level was accepted as $\mathrm{p}<0.05$.

\section{RESULTS}

\section{Pre-angiographic investigations and cause of bleeding}

During the investigations performed before angiography, the presence of active bleeding and/or a bleeding focus were detected in all $18(100 \%)$ patients by endoscopy in 13 cases (72.2\%) and CTA in 10 (55.5\%). The most common pathology causing bleeding was PUD $(n=9 ; 50 \%)$. Other causes of UGIB, pre-procedural investigations, and demographic data of the patients are detailed in Table I.

Table I. Demographic data and clinical parameters of 18 patients with acute upper gastrointestinal bleeding treated by transarterial embolization

\begin{tabular}{|c|c|}
\hline Variable & $\mathrm{n}(\%)$ \\
\hline \multicolumn{2}{|l|}{ Sex } \\
\hline Male & $13(72.2)$ \\
\hline Female & $5(27.8)$ \\
\hline \multicolumn{2}{|l|}{ Age (years) (range: 33-68) } \\
\hline$\leq 60$ & $12(66.7)$ \\
\hline$>60$ & $6(33.3)$ \\
\hline \multicolumn{2}{|l|}{$\begin{array}{l}\text { Units of blood transfusion } 24 \text { hours before angiography } \\
\text { (range: } 2-5 \text { ) }\end{array}$} \\
\hline$<4$ & $4(22.2)$ \\
\hline$\geq 4$ & $14(77.8)$ \\
\hline \multicolumn{2}{|l|}{ Comorbidity } \\
\hline Cardiovascular disease (IHD, HT) & $12(66.7)$ \\
\hline Malignity & $5(27.8)$ \\
\hline Metabolic disease (DM and DLP) & $11(61.1)$ \\
\hline Unknown & $4(22.2)$ \\
\hline \multicolumn{2}{|c|}{ Serum $\mathrm{Hb}$ level on the day of bleeding $(\mathrm{g} / \mathrm{dL})$ (range: 6-11) } \\
\hline$<8$ & $13(72.2)$ \\
\hline$\geq 8$ & $5(27.8)$ \\
\hline \multicolumn{2}{|l|}{ Coagulation } \\
\hline Normal & $14(77.8)$ \\
\hline Coagulopathy (prolonged PT, PTT, or INR) & $2(11.7)$ \\
\hline Thrombocytopenia (platelet count $<50,000 / \mathrm{mm}^{3}$ ) & $2(11.7)$ \\
\hline \multicolumn{2}{|l|}{ Hemodynamic instability } \\
\hline No & $2(11.1)$ \\
\hline Yes & $16(88.9)$ \\
\hline \multicolumn{2}{|l|}{ Investigation before TAE within seven days } \\
\hline Not performed & - \\
\hline Negative CTA & $7(38.9)$ \\
\hline Negative endoscopy & $4(22.2)$ \\
\hline Positive CTA & $10(55.5)$ \\
\hline Positive endoscopy & $13(72.2)$ \\
\hline \multicolumn{2}{|l|}{ Etiology of bleeding } \\
\hline Peptic ulcer disease & $9(50)$ \\
\hline Tumor bleeding & $5(27.8)$ \\
\hline Traumatic or iatrogenic & $4(22.2)$ \\
\hline
\end{tabular}

DM: diabetes mellitus, DLP: dyslipidemia, IHD: ischemic heart disease, HT: hypertension, Hb: hemoglobin, PT: prothrombin time, PTT: partial thromboplastin time, INR: international normalized ratio, TAE: transarterial 


\section{Angiography and embolization}

Positive angiography findings were present in 13 (72.2\%) of patients with acute UGIB. In order of frequency, these findings were pseudoaneurysms in seven patients (38.9\%), tumor staining in four (22.2\%), tumor staining and a pseudoaneurysm in one $(5.5 \%)$, and contrast extravasation in one (5.5\%). In the remaining five (27.8\%) patients, no positive findings were detected on angiography. The focus of UGIB most frequently had a gastric origin fed by the celiac artery $(\mathrm{n}=13 ; 72.2 \%)$. The most commonly embolized artery was the gastroduodenal artery (GDA) $(\mathrm{n}=11 ; 61.1 \%)$. Pathologies causing bleeding, embolized arteries, and embolization methods used are detailed in Table II.

Table II. Angiography findings, treatments applied, and clinical outcomes of 18 patients with acute gastrointestinal bleeding

\begin{tabular}{|c|c|c|c|c|c|c|}
\hline $\begin{array}{l}\text { Patient } \\
\text { Number }\end{array}$ & $\begin{array}{l}\text { Age / } \\
\text { sex }\end{array}$ & Etiology & Angiography findings & Endovascular treatment & Clinical outcome & $\begin{array}{l}\text { Follow-up } \\
\text { (30-day) } \\
\text { mortality }\end{array}$ \\
\hline 1 & $58 / \mathrm{F}$ & PUD & - & $\begin{array}{l}\text { Blind embolization of GDA } \\
\text { (PVA and coil) }\end{array}$ & BS and NFB & - \\
\hline 2 & $60 / \mathrm{F}$ & Gastric lymphoma & Tumor staining & $\begin{array}{l}\text { Blind embolization of GDA } \\
\text { (PVA and coil) }\end{array}$ & BS and NFB & $\begin{array}{l}\text { Died on day, due to } \\
\text { AMI }\end{array}$ \\
\hline 3 & $63 / \mathrm{M}$ & PUD & $\begin{array}{l}\text { Superior pancreaticoduodenal } \\
\text { artery pseudoaneurysm }\end{array}$ & $\begin{array}{l}\text { Coil embolization of the } \\
\text { aneurysm }\end{array}$ & BS and NFB & - \\
\hline 4 & $57 / \mathrm{M}$ & Periampullary tumor & Tumor staining & $\begin{array}{l}\text { Blind embolization of GDA } \\
\text { (PVA and coil) }\end{array}$ & BS and NFB & $\begin{array}{l}\text { Died on day } 3 \text { due to } \\
\text { underlying malignancy }\end{array}$ \\
\hline 5 & $60 / \mathrm{F}$ & Periampullary tumor & $\begin{array}{l}\text { Tumor staining and } \\
\text { microaneurysms in the } \\
\text { branches of GDA }\end{array}$ & $\begin{array}{l}\text { Coil embolization of GDA and } \\
\text { PVA embolization of the tumor }\end{array}$ & BS and NFB & $\begin{array}{l}\text { Died on day } 4 \text { due to } \\
\text { multiorgan failure }\end{array}$ \\
\hline 6 & $60 / \mathrm{M}$ & PUD & GDA pseudoaneurysm & Coil embolization & BS and NFB & - \\
\hline 7 & $65 / \mathrm{M}$ & PUD & - & $\begin{array}{l}\text { Blind embolization of GDA } \\
\text { (PVA and coil) }\end{array}$ & BS and NFB & - \\
\hline 8 & $68 / \mathrm{M}$ & Gastric tumor & Tumor staining & $\begin{array}{l}\text { PVA embolization of the left } \\
\text { gastric artery }\end{array}$ & BS and NFB & $\begin{array}{l}\text { Died on day } 10 \text { due to } \\
\text { underlying malignancy }\end{array}$ \\
\hline 9 & $56 / \mathrm{M}$ & PUD & - & $\begin{array}{l}\text { Blind embolization of GDA } \\
\text { (PVA and coil) }\end{array}$ & BS and NFB & - \\
\hline 10 & $58 / \mathrm{M}$ & Gastric tumor & Tumor staining & $\begin{array}{l}\text { PVA embolization of the right } \\
\text { gastric artery }\end{array}$ & BS and NFB & - \\
\hline 11 & $48 / \mathrm{F}$ & PUD & - & $\begin{array}{l}\text { Blind embolization of GDA } \\
\text { (PVA and coil) }\end{array}$ & $\begin{array}{l}\text { Recurrent bleeding } 16 \\
\text { hours later; underwent } \\
\text { surgical revision }\end{array}$ & $\begin{array}{l}\text { Died on day } 2 \text { due to } \\
\text { uncontrolled bleeding }\end{array}$ \\
\hline 12 & $46 / \mathrm{M}$ & PUD & GDA pseudoaneurysm & Coil embolization & \begin{tabular}{|l|} 
Recurrent bleeding 24 \\
hours later; underwent \\
surgical revision
\end{tabular} & $\begin{array}{l}\text { Died on day } 10 \text { due to } \\
\text { abdominal sepsis }\end{array}$ \\
\hline 13 & $44 / \mathrm{M}$ & $\begin{array}{l}\text { Following PTBD } \\
\text { (iatrogenic) } \\
\end{array}$ & $\begin{array}{l}\text { Left hepatic artery } \\
\text { pseudoaneurysm }\end{array}$ & Coil embolization & BS and NFB & - \\
\hline 14 & $50 / \mathrm{M}$ & PUD & GDA pseudoaneurysm & Coil embolization & BS and NFB & - \\
\hline 15 & $55 / \mathrm{M}$ & PUD & - & $\begin{array}{l}\text { Blind embolization of GDA } \\
\text { (PVA and coil) }\end{array}$ & BS and NFB & - \\
\hline 16 & $33 / \mathrm{M}$ & Trauma & $\begin{array}{l}\text { Contrast extravasation from } \\
\text { the left gastro-epiploic artery }\end{array}$ & PVA and coil embolization & BS and NFB & - \\
\hline 17 & $54 / \mathrm{M}$ & $\begin{array}{l}\text { Following PAD } \\
\text { (iatrogenic) }\end{array}$ & $\begin{array}{l}\text { Left hepatic artery } \\
\text { pseudoaneurysm }\end{array}$ & Coil embolization & $\begin{array}{l}\text { Recurrent bleeding } 12 \mathrm{~h} \\
\text { later; underwent covered } \\
\text { stent-graft placement }\end{array}$ & - \\
\hline 18 & $55 / \mathrm{F}$ & $\begin{array}{l}\text { Following } \\
\text { cholecystectomy } \\
\text { (iatrogenic) } \\
\end{array}$ & Right hepatic artery aneurysms & (covered stent-graft placement) & BS and NFB & - \\
\hline
\end{tabular}

GDA: gastroduodenal artery, PAD: percutaneous abscess drainage, PTBD: percutaneous transhepatic biliary drainage, PVA: polyvinyl alcohol particles, AMI: acute myocardial infarction, PUD: peptic ulcer disease, BS and NFB: Bleeding stopped and no further bleeding. The sizes of the PVA particles used were 355-500, 500-710 and 710-1,000 microns 
Due to hepatic artery pseudoaneurysms (HAPs), three (16.7\%) patients developed hemobilia and UGIB after liver transplantation, cholecystectomy, and percutaneous transhepatic biliary drainage (PTBD) catheterization, respectively. Although, the patient that developed HAP after the liver transplant underwent embolization of the aneurysm sac by coil, rebleeding occurred after 12 hours. During the second angiography, a covered stent-graft was placed while preserving the hepatic artery. No mortality or complications developed during the 30 -day follow-up. In the second patient, the $5 \times 9.6 \mathrm{~cm}$ narrownecked giant aneurysm that developed after cholecystectomy was treated with a stent-graft (Figure 1). The third patient developed HAP due to the PTBD catheter placed in the left lobe in another hospital, but a diagnosis could not be made there despite the massive bleeding from the catheter site and upper gastrointestinal tract. This patient was referred to our center with hemodynamic instability, and the bleeding focus could not be determined on endoscopy or the first CTA examination. Thereupon, the catheter in the left lobe was removed, and the repeat CTA revealed a pseudoaneurysms in the left hepatic artery; thus, the patient was immediately taken to the interventional radiology unit. Embolization was performed with microcoils upon the detection of the left HAP in the selective hepatic arteriogram. The patient was discharged after observing no bleeding or complication during the follow-up (Figure 2).

In five $(27.8 \%)$ patients with peptic ulcers, the finding that caused bleeding (extravasation or pseudoaneurysm) could not be detected by angiography. Following the administration of PVA particles through GDA to these patients, blind embolization was performed with the sandwich method using a coil. No retrograde filling was detected in the control angiography performed from SMA after embolization. Surgical revision was performed in one of these patients due to rebleeding that occurred after 16 hours. However, on the second day, mortality developed due to uncontrollable bleeding. No signs of bleeding or ischemia developed in the follow-up of the remaining four patients with peptic ulcers.
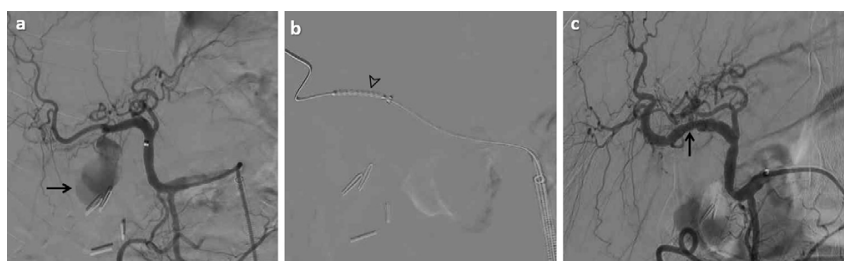

Figure 1. Digital subtraction angiography images of a 55-year-old patient that developed a hepatic artery pseudoaneurysm following cholecystectomy. a Angiography before the procedure reveals a $5 \times 9.6 \mathrm{~cm}$-sized pseudoaneurysm sac (arrow) with a short neck originating from the right hepatic artery. $b$ Angiography showing the placement of a covered stent-graft (arrow head). $c$ disappearance of the aneurysm and continuation of blood flow through the covered stent-grafts (arrow) placed by preserving the hepatic artery.

For the pseudoaneurysms originating from GDA $(n=3 ; 16.7 \%)$ and the superior pancreatic-duodenal artery $(n=1 ; 5.5 \%)$ due to peptic ulcers, embolization was performed by coil (Figure 3 ).
One of these patients required surgery due to rebleeding after 24 hours. Mortality caused by sepsis occurred on the $10^{\text {th }}$ day in the follow-up of this patient.
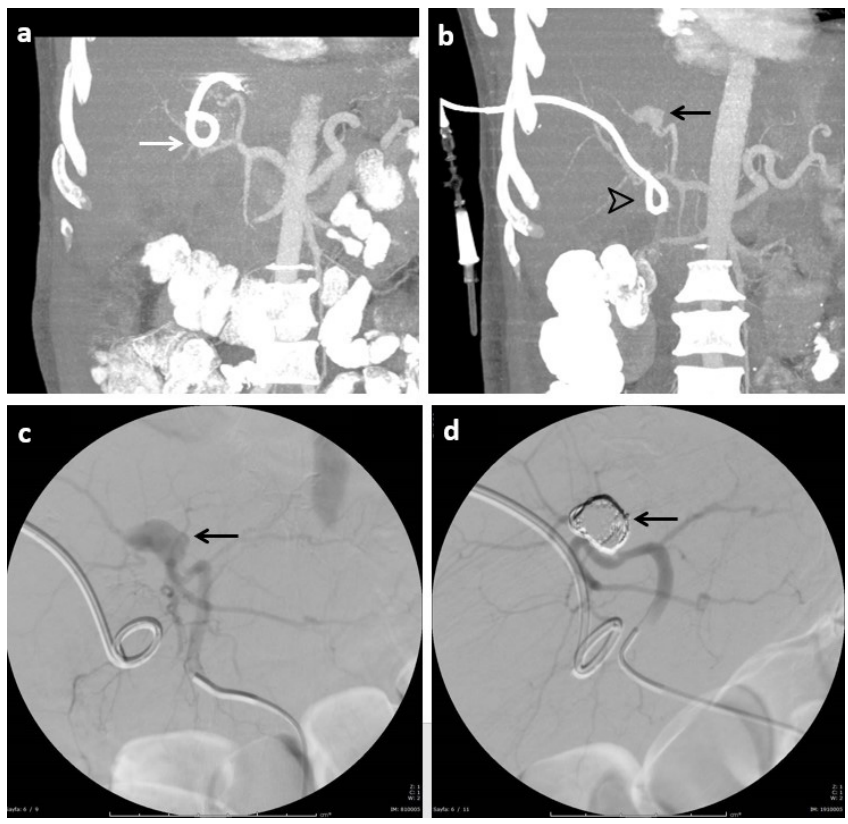

Figure 2. Images of an iatrogenic hepatic artery pseudoaneurysm that developed after PTBD performed due to distal common bile cancer. a The maximum intensity projection image shows no hepatic artery aneurysm when the PTBD catheter (white arrow) is in the biliary duct in the left lobe. $b$ Following the removal of the catheter and placement of a different catheter (arrow head) from the right side, a $2.4 \times 1.3 \mathrm{~cm}$ pseudoaneurysm sac originating from the left hepatic artery (black arrow) appears. $c$ Pre-procedural hepatic arteriography shows the left hepatic artery pseudoaneurysm (arrow). $d$ Post-embolization angiography shows the coil-packed and embolized pseudoaneurysm (arrow).

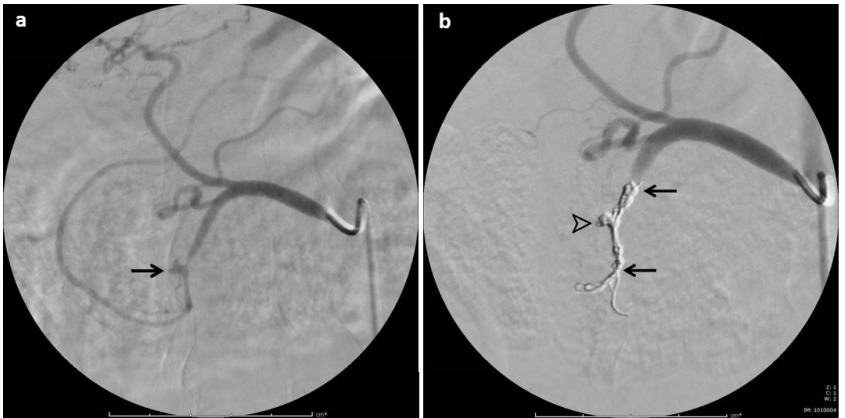

Figure 3. Upper gastrointestinal bleeding in a 50-year-old male with bleeding peptic ulcer and unsuccessful endoscopic hemostasis. a Digital subtraction angiography images reveal the pseudoaneurysm in the gastroduodenal artery (arrow). $b$ The images also show that the pseudoaneurysm in the gastroduodenal artery, which was the source of bleeding, has been embolized by coil (arrow head). It is also shown that the proximal and distal of the artery have been embolized by microcoil using the sandwich technique to prevent rebleeding (arrows). 

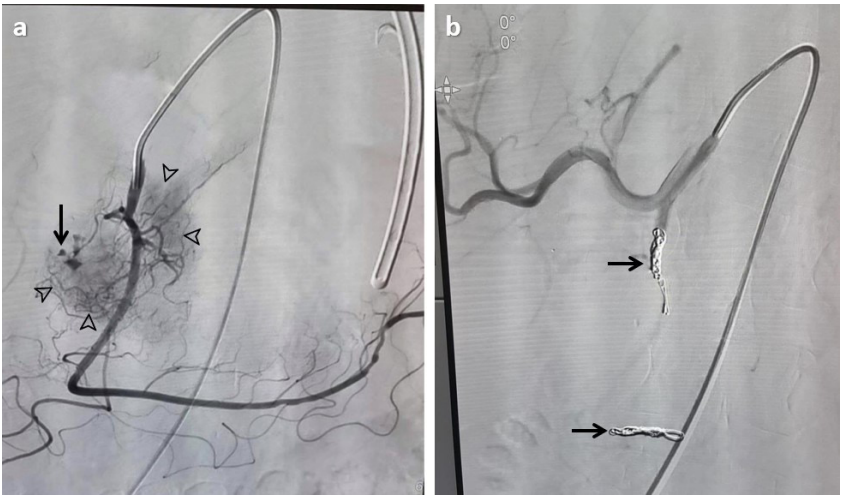

Figure 4. Digital subtraction angiography (DSA) images of a 60-year-old woman with a periampullary tumor. a Pre-embolization selective DSA image shows tumor staining (arrowheads) and microaneurysms (arrow) originating from the gastroduodenal artery. $b$ Post-embolization control DSA image shows that the proximal and distal of the artery have been embolized by coil using the sandwich technique to prevent antegrade and retrograde filling (arrows). Polyvinyl alcohol particles were given before coil embolization.

Various embolizing agents were used to occlude the artery feeding the bleeding tissue. In most cases ( $n=9 ; 50 \%)$, coils and PVA particles were used in combination. This was followed by the use of coils alone $(n=6 ; 33.3 \%)$. Tumor staining and microaneurysm were detected in one of the five $(27.8 \%)$ patients with tumorous bleeding, and embolization was performed in this patient using the coil and PVA particles combination (Figure 4).

Technical success was achieved in all 18 (100\%) patients during the TAE procedure. Catheterization was not possible with a standard femoral introducer in two $(11.1 \%)$ patients due to arterial tortuosity but the procedure was successfully performed using long femoral guiding sheaths in these cases.

After excluding the four patients (22.2\%), who died due to nonUGIB causes (multiorgan failure, underlying malignancy, and acute myocardial infarction) within 30 days of hospitalization (based on the definition of clinical success as the presence of no rebleeding for at least 30 days following TAE), the clinical success rate was calculated as $78.5 \%$ (11/14 patients). As shown in the flow diagram in Figure 5, of the three (16.7\%) patients with clinical failure that developed recurrent bleeding within the first 24 hours, two diagnosed with PUD (11.1\%) required surgical intervention and one diagnosed with HAP (5.5\%) underwent a second angiography.

Table III shows the comparison of clinical success rates according to the causes of bleeding and the types of endovascular treatment performed.

The 30-day mortality rate for all causes was 6/18 (33.3\%). Minor complications (non-flow-limiting arterial dissection at the puncture site) occurred in two (11.1\%) patients due to the procedure. No major complications occurred in any of the patients.

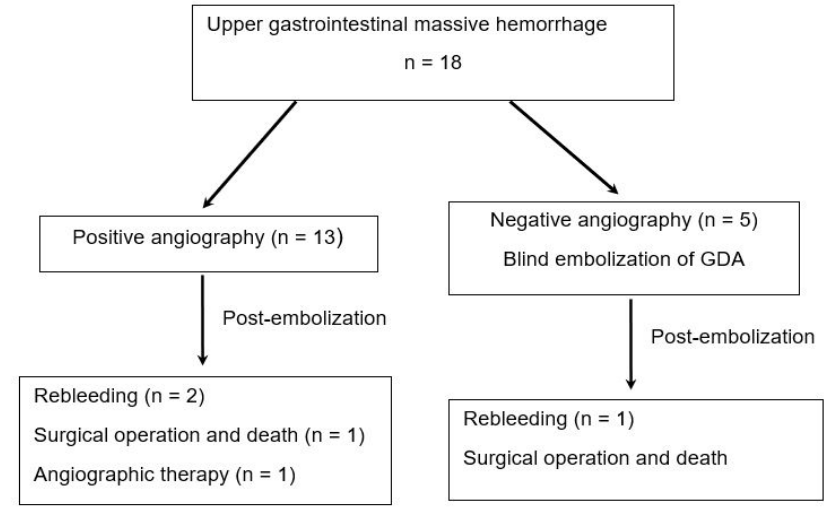

Figure 5. Flow diagram of the patients included in the study

Table III. Comparison of clinical success rates according to the causes of bleeding and endovascular treatment types

\begin{tabular}{|c|l|l|l|}
\hline Etiology of bleeding & Total, $\mathbf{n}(\%)$ & $\begin{array}{c}\text { Clinical } \\
\text { success, } \mathbf{n} \\
(\%)\end{array}$ & p value \\
\hline Peptic ulcer disease & $9(50)$ & $7(77.8)$ & \multirow{2}{*}{0.505} \\
\cline { 1 - 3 } Tumor bleeding & $5(27.8)$ & $5(100)$ & \\
\cline { 1 - 3 } Traumatic or iatrogenic & $4(22.2)$ & $3(75)$ & \multirow{2}{*}{0.485} \\
\hline PVA and coil embolization & $9(50)$ & $8(78.9)$ & \\
\hline Coil embolization & $6(33.3)$ & $4(66.7)$ & \\
\hline PVA embolization & $2(11.1)$ & $2(100)$ & \\
\hline Covered stent-graft placement & $1(5.5)$ & $1(100)$ & \\
\hline
\end{tabular}

$p$ value $<0.05$ at $95 \%$ confidence interval is significant

\section{DISCUSSION}

In this study, the technical and clinical success rates of TAE performed following acute UGIB were $100 \%$ and $78.5 \%$, respectively. There were no cases in which TAE technically failed. This is in agreement with previous studies reporting the technical success rate of TAE to be over 90\% [7-9]. These high success rates can be attributed to the development of endovascular devices with the advancements in technology, growing experience of IRs, and the combined use of embolic agents [10]. Researchers stated that technical failure occurred most frequently due to difficult vascular anatomies, arterial dissection, vasospasm, false negatively read angiogram, multiple 
bleedings, and tumorous bleeding $[9,11,12]$. In our study, this problem was resolved using a long femoral guiding sheath only in two (11.1\%) cases for which selective catheterization was not possible due to arterial tortuosity.

With transarterial embolization, $89-98 \%$ of initial bleeding can be taken under control. The clinical success rate of TAE was found to be different in previous research (52-98\%) while most showing success rates of 70 to $80 \%$ [7-9]. In all patients in the current study, bleeding was successfully controlled at the first stage using the TAE method. Similar to previous studies, a high clinical success rate of $78.5 \%$ was achieved. Among the cases of clinical failure, two diagnosed with PUD experienced rebleeding shortly after the procedure, and thus underwent surgical revision; however, mortality developed in both due to uncontrolled bleeding and abdominal sepsis. The third case of clinical failure presented with the rebleeding of the aneurysm that had been caused by HAP and embolized by coil after liver transplantation. HAP usually occurs as a result of surgical and interventional procedures applied to the biliary tract or develops secondary to trauma. Today, parallel to the increase in percutaneous interventions for the biliary tract, there is an increase in the incidence of pseudoaneurysms observed [13]. Tessier et al., reported that among 153 patients with HAPs, the causes of these pseudoaneurysms in order of frequency were PTBD (31\%), laparoscopic cholecystectomy (28\%), open cholecystectomy (13\%), and percutaneous liver biopsy (7\%) [14]. In addition, if hemobilia, a rare cause of UGIB that occurs due to HAP rupture is not treated immediately, it may result in mortality [9]. All HAP cases in the current study had iatrogenic causes and presented to the clinic with hemobilia. As in the patient that was referred to our center with a delayed diagnosis in a hemodynamically unstable condition, a PTBD catheter can hide existing pseudoaneurysms in some cases and cause delay in treatment. In such cases, investigations performed after the removal of the catheter can be diagnostic. In light of all this information, patients who have undergone liver interventions and developed UGIB should be promptly taken to the angiography unit even if their endoscopy findings are negative. Thus, serious complications, such as HAP, which can result in massive bleeding and mortality, can be treated quickly and safely by TAE.

In this study, PUD (50\%) was the most common cause of bleeding, which is consistent with the literature [9]. This was followed by tumorous bleeding and iatrogenic causes. The majority of patients with positive angiography findings had pseudoaneurysms. Tétreau et al., reported that $17 \%$ of gastrointestinal bleeding occurred due to pseudoaneurysms, and $91 \%$ of these cases were successfully treated by TAE [15]. In the current study, pseudoaneurysms were detected at a rate of $38.9 \%$ on angiography, and embolization was performed. Despite technical success in all patients, mortality related to rebleeding occurred in one case. Nevertheless, previous authors also emphasized that TAE could be successfully applied in patients who develop massive bleeding as a result of splanchnic artery pseudoaneurysms [16].
Angiographic findings of bleeding (contrast extravasation, vascular blush, pseudoaneurysms, or arterial spasm) may not always be evident in massive UGIB. In such cases, blind embolization can be performed on the arteries feeding the bleeding focus. Although blind embolization is controversial, most researchers argue that this procedure should be performed based on endoscopic findings [9, 10]. Aina et al., [17], Loffroy et al., [18], and Padia et al. [19] performed blind embolization of GDA using coils in those with and without positive angiography findings in their case series. Although, the negative extravasation group had a higher incidence of comorbidities, such as respiratory distress, none of these researchers found any difference between the two groups in terms of the treatment success rate and short-term outcome (30-day mortality). However, the sandwich technique, in which the proximal and distal of GDA are filled during the blind embolization procedure, is reported to prevent retrograde filling that may result from SMA [9, 17-19]. In our study, UGIB was taken under control, and hemostasis was achieved in the first stage in all five $(27.8 \%)$ patients, in whom we performed blind embolization with the sandwich technique. Only one patient had rebleeding. Based on this information and the results of our study, it is possible to state that blind embolization is an effective treatment for hemostasis control when performed with the appropriate technique.

Various embolic agents, such as gelatin sponges, metallic coils, PVA, glue, N-butyl cyanoacrylate, and autologous blood clots can be used in the TAE procedure $[3,9,10]$. In this study, the combination of coil and PVA particles was most commonly used for embolization. During the procedure, attention was paid to placing the coils close to the bleeding artery as possible, which was supplemented by PVA to embolize collaterals with bleeding potential. Recurrent bleeding being seen in only one patient shows that the combined use of PVA with a coil results in a successful procedure. Although, there are a few researchers who applied coils alone in the embolization of GDA and pancreaticoduodenal arteries, it is generally recommended to use coils together with gelatin sponge or PVA to successfully achieve distal levels of hemostasis. Despite the low cost and easy availability of gelatin sponge, due to its resorbable nature, it leads to higher recurrent bleeding rates compared to non-resorbable PVA. Furthermore, studies have shown that the success rate of using gelatin sponge alone is very low (62\%), and therefore it should be avoided to achieve long-term hemostasis [9]. Aina et al., [17] and Loffroy et al., [18] showed a relationship between the use of coils alone and incidence of recurrent bleeding, especially in patients with coagulopathy, and emphasized the advantages of using it in combination with PVA or gelatin sponge could not be overlooked. In the current study, coils were used alone only in the treatment of six patients with pseudoaneurysms (33.3\%), and clinical success was achieved in all but two patients. In the patients with a liver transplant that developed bleeding, embolization of the parent artery was avoided due to the risk of liver failure, and a stent-graft was placed. Mortality and morbidity did not develop during the short-term follow-up (30 days). In such cases, specific endovascular treatments, such as filling the pseudoaneurysm sac with a coil or placing a covered 
stent can provide favorable results in maintaining the patency of the parent artery [9]. To date, only a few cases of endovascular covered stent-graft repair have been described during acute bleeding in liver transplant patients. The use of a covered stent with a stiff structure is a technically difficult method due to the tortuosity of the transplanted hepatic artery [20]. Given the success rates observed in other reports and in our own experience, we believe that the proper and safe endovascular placement of a covered stent-graft may be a good alternative to open surgery because this approach can simultaneously control active bleeding and reconstruct HAP after liver transplantation. Therefore, it should be considered as a less morbid approach in high-risk patients.

In this study, the total 30 -day mortality rate was $33.3 \%$, which is similar to previous studies (20-46\%) [9, 17-19]. Following TAE performed with technical success, most in-hospital mortality (4/6) occurred for non-UGIB causes, but it was as a result of the deterioration of patients' comorbidities. This is in agreement with the reports in the literature [9]. The variability of the mortality rates among the studies in the literature further emphasizes the importance of individual expertise and center experience. In addition, although mortality rates seem to be high, patients undergoing TAE generally consist of an older age population with a high incidence of comorbidities, who are often unable to bear the surgical burden. Therefore, providing bleeding control with TAE seems to reduce the incidence of laparotomy, and thus indirectly decrease morbidity and mortality in patients.

\section{Limitations}

This study had certain limitations, such as its retrospective design and absence of randomization. The current study reveals that different embolization methods and bleeding etiology have no effect on clinical success. However, the small number of patients between the groups may not give reliable results for comparison. This is among the limitations of our study. Therefore, large population-based, prospective, and, if possible, multicenter studies are needed to better explain the best initial management in patients with active UGIB and to elucidate the variables that may affect the treatment outcome.

\section{Conclusions}

TAE is a safe and effective treatment method with high technical and clinical success rates in the treatment of life-threatening acute non-varicose UGIB. It is concluded that in cases with endoscopy-refractory acute massive UGIB, TAE should be promptly performed by experienced IRs, and a multidisciplinary team is required for the pre - and post-procedural management of patients.

\section{Compliance with Ethical Standards}

Ethical approval: All procedures performed in this study were in accordance with the ethical standards of institutional and/ or national research committees and with the 1964 Declaration of Helsinki and its later amendments or comparable ethical standards. The research project was approved by Harran University, Faculty of Medicine Institutional Review Board (approval number: 20/08/12, 27.04.2020). Informed consent was obtained from all individual participant included in the study.

Funding: The study was not supported by any funds.

Conflict of Interest: The authors have no conflicts of interest to declare.

Authors' Contrubitions: Concept and Design: MT and MK, Resources: MT and MK, Materials: MK, Data Collection and processing: MK, Analysis and interpretation: MT and MK, Literature search: MT and MK, Writing the article article: MT and MK, Critical Review: MT and MK. Both authors read and approved the final version of this article.

\section{REFERENCES}

[1] Bua-Ngam C, Norasetsingh J, Treesit T, et al. Efficacy of emergency transarterial embolization in acute lower gastrointestinal bleeding: A single-center experience. Diagn Interv Imaging 2017;98:499-505. doi.org/10.1016/j. diii.2017.02.005.

[2] Augustin AM, Fluck F, Bley T, Kickuth R. Endovascular therapy of gastrointestinal bleeding. RöFo Fortschritte Auf Dem Gebiet Der Rontgenstrahlen Und Der Bildgeb Verfahren 2019;191:1073-82. doi.org/10.1055/a-0891-1116.

[3] Chang WC, Liu CH, Hsu HH, et al. Intra-arterial treatment in patients with acute massive gastrointestinal bleeding after endoscopic failure: Comparisons between positive versus negative contrast extravasation groups. Korean J Radiol 2011;12:568-78. doi.org/10.3348/kjr.2011.12.5.568.

[4] Loffroy RF. Recent advances in endovascular techniques for management of acute nonvariceal upper gastrointestinal bleeding. World J Gastrointest Surg 2011;3:89. doi. org/10.4240/wjgs.v3.i7.89.

[5] Pannatier M, Duran R, Denys A, Meuli R, Zingg T, Schmidt S. Characteristics of patients treated for active lower gastrointestinal bleeding detected by CT angiography: Interventional radiology versus surgery. Eur J Radiol 2019;120:108691. doi.org/10.1016/j.ejrad.2019.108691.

[6] Köhler G, Koch OO, Antoniou SA, et al. Relevance of Surgery after Embolization of Gastrointestinal and Abdominal Hemorrhage. World J Surg 2014;38:2258-66. doi.org/10.1007/ s00268.014.2570-7.

[7] Loffroy R, Rao P, Ota S, De Lin M, Kwak B-K, Geschwind J-F. Embolization of acute nonvariceal upper gastrointestinal hemorrhage resistant to endoscopic treatment: results and predictors of recurrent bleeding. Cardiovasc Intervent Radiol 2010;33:1088-100. doi.org/10.1007/s00270.010.9829-7.

[8] Loffroy R, Guiu B, Mezzetta L, et al. Short - and long-term results of transcatheter embolization for massive arterial hemorrhage from gastroduodenal ulcers not controlled by endoscopic hemostasis. Can J Gastroenterol 2009;23:115-20. doi.org/10.1155/2009/795460.

[9] Loffroy R, Favelier S, Pottecher P, et al. Transcatheter arterial embolization for acute nonvariceal upper gastrointestinal bleeding: Indications, techniques and outcomes. Diagn Interv Imaging 2015;96:731-44. doi.org/10.1016/j.diii.2015.05.002. 
[10] Muhammad A, Awais M, Sayani R, et al. Empiric transcatheter arterial embolization for massive or recurrent gastrointestinal bleeding: ten-year experience from a single tertiary care center. Cureus 2019;11:e4228. doi.org/10.7759/cureus.4228.

[11] Hongsakul K, Pakdeejit S, Tanutit P. Outcome and predictive factors of successful transarterial embolization for the treatment of acute gastrointestinal hemorrhage. Acta Radiol 2014;55:186-94. doi.org/10.1177/028.418.5113494985.

[12] Gillespie CJ, Sutherland AD, Mossop PJ, Woods RJ, Keck JO, Heriot AG. Mesenteric embolization for lower gastrointestinal bleeding. Dis Colon Rectum 2010;53:1258-64. doi. org/10.1007/DCR.0b013e3181e10e90.

[13] An JY, Lee JS, Kim DR, et al. Coil embolization of ruptured intrahepatic pseudoaneurysm through percutaneous transhepatic biliary drainage. Yeungnam Univ J Med 2018;35:109-13. doi.org/10.12701/yujm.2018.35.1.109.

[14] Tessier DJ, Fowl RJ, Stone WM, et al. Iatrogenic hepatic artery pseudoaneurysms: an uncommon complication after hepatic, biliary, and pancreatic procedures. Ann Vasc Surg 2003;17:663-9. doi.org/10.1007/s10016.003.0075-1.

[15] Tétreau R, Beji H, Henry L, Valette P-J, Pilleul F. Arterial splanchnic aneurysms: Presentation, treatment and outcome in 112 patients. Diagn Interv Imaging 2016;97:81-90. doi. org/10.1016/j.diii.2015.06.014.
[16] Dohan A, Eveno C, Dautry R, et al. Role and effectiveness of percutaneous arterial embolization in hemodynamically unstable patients with ruptured splanchnic artery pseudoaneurysms. Cardiovasc Intervent Radiol 2015;38:86270. doi.org/10.1007/s00270.014.1002-2.

[17] Aina R, Oliva VL, Therasse É, et al. Arterial embolotherapy for upper gastrointestinal hemorrhage: outcome assessment. J Vasc Interv Radiol 2001;12:195-200. doi.org/10.1016/S10510443(07)61825-9.

[18] Loffroy R, Guiu B, D'Athis P, et al. Arterial embolotherapy for endoscopically unmanageable acute gastroduodenal hemorrhage: Predictors of early rebleeding. Clin Gastroenterol Hepatol 2009;7:515-23. doi.org/10.1016/j.cgh.2009.02.003.

[19] Padia SA, Geisinger MA, Newman JS, Pierce G, Obuchowski NA, Sands MJ. Effectiveness of coil embolization in angiographically detectable versus non-detectable sources of upper gastrointestinal hemorrhage. J Vasc Interv Radiol 2009;20:461-6. doi.org/10.1016/j.jvir.2009.01.006.

[20] Zhang J, Khalifeh A, Santini-Dominguez R, et al. Endovascular reconstruction of the hepatic arterial system for the management of mycotic pseudoaneurysm in a liver transplant patient. Ann Vasc Surg 2019;61:473.e7-473.e11. doi.org/10.1016/j.avsg.2019.05.060. 